\section{Sintomas articulares crônicos em adultos de Pelotas, Rio Grande do Sul, Brasil: prevalência e determinantes}

\author{
Chronic joint symptoms in adults from Pelotas, \\ Rio Grande do Sul State, Brazil: prevalence \\ and determinants
}

\author{
1 Universidade Federal de \\ Pelotas, Pelotas, Brasil. \\ Correspondência \\ V. R. L. Silva \\ Universidade Federal \\ de Pelotas. \\ Rua Santa Cruz 2439, \\ apto. 404, Pelotas, $R S$ \\ 96015-710, Brasil. \\ veralopess@yahoo.com.br
}

\begin{abstract}
With the objective of estimating the prevalence of chronic joint symptoms and their determinants, a cross-sectional study was conducted with 2,953 adults 20 years or older in Pelotas, Rio Grande do Sul State, Brazil. Pain, swelling, and stiffness in joints were investigated, plus demographic, socioeconomic, and health-related information. Multivariate analysis was performed with Poisson regression. Overall prevalence of chronic joint symptoms was $36.5 \%$ (42.4\% in women and $28.7 \%$ in men). The threefold presence of pain, swelling, and stiffness in joints was $14.1 \%$ and $5.5 \%$ in women and men, respectively. Chronic joint symptoms showed a linear association with increasing age (PR: 2.9; 95\%CI: 2.4-3.5), body mass index (PR: 1.6; 95\%CI: 1.3-2.0), and less schooling (PR: 1.5; 95\%CI: 1.3-1.8). Only 6\% of men and $18 \%$ of women had a medical diagnosis of arthritis. Overall prevalence rates for limitations in activities of daily living and work secondary to chronic joint symptoms were $15 \%$ and $21 \%$, respectively. Young people and men represent the majority of individuals with under-diagnosed chronic joint symptoms.
\end{abstract}

Arthralgia; Arthritis; Rheumatic Diseases; Joints
Vera Regina Lopes da Silva 1 Ana Maria Baptista Menezes 1 Ricardo Bica Noal 1

\section{Introdução}

Artrite ou reumatismo são termos usados corriqueiramente para designar uma gama de enfermidades muito distintas entre si, mas que apresentam como um dos alvos principais a articulação sinovial. A Organização Mundial da Saúde (OMS) declarou que os anos de 2000 a 2010 constituiriam a "Década do Osso e da Articulação” (http://www.boneandjointdecade. org) e com isso estimulou o desenvolvimento de pesquisas nesta área em razão do aumento da prevalência e do impacto econômico e social das doenças reumatológicas.

Como o processo inflamatório articular se expressa geralmente por dor, edema, rigidez matinal e limitação da mobilidade na topografia da articulação comprometida, alguns pesquisadores 1,2,3 têm avaliado a prevalência de artrite na comunidade por intermédio do relato de sintomas articulares crônicos ou do diagnóstico médico referido.

Um estudo realizado em todo o território norte-americano, com adultos não institucionalizados, com idade $\geq 18$ anos, evidenciou que $33 \%$ (IC95\%: 32,7-33,4) dos indivíduos entrevistados apresentavam sintomas articulares crônicos ou artrite 4 . No Brasil, em um estudo de âmbito nacional, representativo da população com mais de 60 anos, artrite foi a segunda condição auto-referida mais prevalente (37,5\%; IC95\%: 30,0-43,6), atrás somente de hipertensão arterial sistêmica 5 . 
Nessa mesma faixa etária, na cidade de Bambuí, em Minas Gerais, Machado et al. 6 encontraram uma prevalência de $44 \%$ e de $25 \%$ de sintomas articulares crônicos e de diagnóstico médico de reumatismo, respectivamente.

A prevalência de várias doenças reumatológicas, tais como artrite reumatóide, lúpus eritematoso sistêmico e síndrome de Sjögren, é, no mínimo, o dobro no sexo feminino 7. Outros pesquisadores verificaram que as mulheres referem 1,3 a 1,7 vezes mais sintomas articulares crônicos ou artrite do que os homens 4,8 . Além do sexo feminino, outros fatores de risco para sintomas articulares crônicos têm sido demonstrados na literatura. Dentre esses, podem ser citados a presença de outras doenças crônicas, como infarto agudo do miocárdio e acidente vascular cerebral, o aumento da idade e do índice de massa corporal (IMC) e baixa escolaridade 4,6,9. Contrariando o senso comum de que esta é uma enfermidade exclusiva dos idosos, dos quatro milhões de canadenses adultos com diagnóstico médico referido de artrite, $60 \%$ deles estão numa faixa etária economicamente ativa, ou seja, entre $18 \mathrm{e}$ 64 anos ${ }^{8}$. Um estudo conduzido no Reino Unido estimou que a prevalência de incapacidade por doenças reumatológicas é de $82 /$ mil na população com 16 anos ou mais 1 . Uma análise, baseada no censo dos Estados Unidos, representativa da população civil, não institucionalizada e com idade $\geq 18$ anos evidenciou que $22 \%$ dos adultos referem ter algum tipo de incapacidade e, $63 \%$ deste percentual, ou seja, aproximadamente 27,8 milhões de pessoas, estão na faixa etária dos 18 aos 64 anos 10.

Considerando a elevada ocorrência dos sintomas articulares crônicos, sua presença em todas as faixas etárias, sua associação com limitação funcional e laboral, bem como a ausência de dados nacionais para a população abaixo dos 60 anos, este estudo teve como objetivo determinar a prevalência e avaliar fatores associados aos sintomas articulares crônicos em adultos.

\section{Métodos}

Entre outubro de 2007 e janeiro de 2008 foi realizado um estudo transversal, de base populacional, com adultos maiores de 20 anos, residentes na zona urbana de Pelotas, cidade com cerca de 340 mil habitantes, localizada ao sul do Brasil. Por um sistema de consórcio, um grupo de pesquisadores, com projetos independentes, reuniu-se para a coleta de dados com um instrumento único.

Todos os indivíduos elegíveis (idade $\geq 20$ anos), residentes nos domicílios sorteados, res- ponderam a um questionário padronizado e pré-codificado, aplicado por 30 entrevistadoras previamente treinadas, contendo questões referentes aos aspectos demográficos, sócio-econômicos, comportamentais e de saúde.

A variável dependente - sintomas articulares crônicos - foi definida como a resposta afirmativa a pelo menos uma das seguintes perguntas: (1) “ $O(A) \operatorname{Sr}(a)$. teve dor ou dolorimento nas juntas, que durou a maior parte dos dias, por pelos menos um mês e meio?”; (2) "O(A) Sr(a). teve inchaço nas juntas, que durou a maior parte dos dias, por pelos menos um mês e meio?"; e (3) "O(A) Sr(a). teve endurecimento ou dificuldade para mexer as juntas, ao levantar pela manhã, que durou a maior parte dos dias, por pelos menos um mês e meio?". O período recordatório foram os 12 meses que antecederam ao estudo e as questões foram adaptadas do módulo de artrite do Behavioral Risk Factor Surveillance System 11.

Com relação às variáveis independentes, as demográficas utilizadas foram: sexo - categórica dicotômica; idade (em anos completos) - categórica ordinal; e cor da pele (referida pelo entrevistado como branca, negra/parda e outras) - categórica nominal. A variável sócioeconômica foi escolaridade (anos de estudo completos) - categórica ordinal. As variáveis comportamentais incluíram tabagismo (nunca fumou, ex-fumante e fumante atual) - categórica ordinal - e sedentarismo (menos 150 minutos/ semana, segundo a versão curta do Questionário Internacional de Atividades Físicas [IPAQ] 12, que contempla atividades físicas contínuas, com pelo menos dez minutos de duração, na semana anterior à entrevista) - categórica dicotômica. As variáveis de saúde foram: IMC, de acordo com peso e altura auto-referidos (peso normal $\leq 24,9 \mathrm{~kg}$ / $\mathrm{m}^{2}$, sobrepeso $25-29,9 \mathrm{~kg} / \mathrm{m}^{2}$ e obesidade $\geq 30 \mathrm{~kg}$ / $\mathrm{m}^{2}$ ) - categórica ordinal 13; hipertensão arterial sistêmica (definida como a média aritmética de duas aferições da pressão arterial $\geq 140 / 90$ mmHg [monitor de pulso, marca Omron modelo 631; Omron Brasil, São Paulo, Brasil] e/ou uso de medicação anti-hipertensiva) - categórica dicotômica; angina pectoris (definida pelo Questionário Rose 14, aplicado somente aos indivíduos com idade $\geq 40$ anos) - categórica dicotômica; diabetes mellitus (diagnóstico médico referido) - categórica dicotômica; e histórico familiar de artrite ou reumatismo - categórica dicotômica.

Ainda questionou-se sobre diagnóstico médico de artrite ou reumatismo (referido pelo entrevistado) - categórica dicotômica; trabalho braçal e repetitivo (categorizado em nunca reali$\mathrm{za}$, realiza às vezes ou realiza sempre nos últimos 24 meses) - categórica nominal; e a presença de restrição funcional, para atividades de vida diá- 
ria e laboral, secundária a sintomas articulares crônicos. Essas foram definidas como resposta positiva às seguintes questões: (1) "O reumatismo ou estes problemas das juntas atrapalha as suas atividade do dia-a-dia, como se vestir, tomar banho, se pentear ou se alimentar sozinho?"; (2) "O reumatismo ou estes problemas das juntas atrapalha as suas atividade de trabalho, serviço da casa ou estudo?" e categorizada como muita, moderada, pouca ou nenhuma restrição (categórica nominal).

Para o cálculo do tamanho amostral, considerou-se uma prevalência estimada de sintomas articulares crônicos de $25 \%$ e um erro aceitável de 2,5 pontos percentuais. Para avaliar as associações existentes com os fatores de risco, estabeleceu-se um poder de $80 \%$ e um nível de $95 \%$ de confiança para detectar uma razão de prevalência de no mínimo 1,4. Visando compensar eventuais perdas e recusas e para controle dos fatores de confusão, foram acrescidas à amostra calculada $10 \%$ e $15 \%$, respectivamente, resultando em um total de 1.475 indivíduos a serem entrevistados. Prevendo o efeito do delineamento, que pode ocorrer na amostragem por conglomerados, estimamos um acréscimo de $100 \%$, o que determinou um tamanho de amostra de 2.655 indivíduos ou 1.154 domicílios.

Foi adotado um processo de amostragem em dois estágios. De acordo com o último censo demográfico realizado em 2000 pelo Instituto Brasileiro de Geografia e Estatística (IBGE; http:// www.ibge.gov.br), os 408 setores censitários foram listados e as residências estratificadas em ordem crescente segundo a renda média dos chefes de famílias. Posteriormente, foram sorteados 126 setores com probabilidades proporcionais ao tamanho. Por causa do tempo decorrido desde o último censo, fez-se uma recontagem para atualização do número de domicílios por setor. Em cada um deles, por conta da modificação ocorrida - aumento ou diminuição - no número de domicílios em cada um dos setores, 11 domicílios, em média, foram sorteados de forma sistemática, fato que resultou em 1.522 domicílios elegíveis para o estudo. Foram excluídas da amostra as pessoas institucionalizadas e aquelas com deficiência mental.

A digitação dos dados seguiu o procedimento de dupla entrada no programa Epi Info 6.04 (Centers for Disease Control and Prevention, Atlanta, Estados Unidos), com comparação das digitações e análise de consistência entre elas. O controle de qualidade foi realizado pela aplicação de um questionário simplificado, em até 14 dias após a primeira entrevista, em 10\% dos entrevistados sorteados aleatoriamente.
A análise estatística foi realizada no programa Stata 10.0 (Stata Corp., College Station, Estados Unidos) e a significância estatística das associações foram calculadas pelos testes de Wald de heterogeneidade para as variáveis dicotômicas e nominais, e de tendência para as variáveis categóricas ordinais. Utilizou-se regressão de Poisson para estimar as razões de prevalência (RP) e seus respectivos IC95\%. A análise ajustada foi realizada por um modelo hierarquizado e estratificada por sexo, permanecendo no modelo as variáveis independentes que apresentaram associação com sintomas articulares crônicos, com nível de significância $\leq 0,20$. Na análise ajustada, distalmente, ficaram idade e cor da pele; no segundo nível ficaram escolaridade e história familiar de artrite ou reumatismo; no terceiro nível foram contempladas as co-morbidades (hipertensão arterial sistêmica, diabetes mellitus e angina pectoris) e tabagismo e, proximalmente ao desfecho ficaram IMC e sedentarismo.

Todos os indivíduos participantes assinaram um termo de consentimento livre e esclarecido e o projeto foi aprovado de Comitê de Ética da Faculdade de Medicina da Universidade Federal de Pelotas.

\section{Resultados}

O número total de indivíduos elegíveis para este estudo foi de 3.147, e, dentre eles, 194 (6,2\%) foram perdas ou recusas, o que resultou em 2.953 adultos entrevistados. A análise descritiva (Tabela 1) demonstra que a maioria dos indivíduos foi do sexo feminino $(56,9 \%)$, de cor da pele branca $(75,6 \%)$ e com até oito anos de escolaridade (54,8\%). A média de idade e o desvio-padrão (DP) para as mulheres foi de 45,4 (DP $\pm 17,3$ ) anos e para os homens foi de 43,4 (DP $\pm 16,2)$ anos. Mais da metade dos indivíduos possuía histórico familiar de artrite ou reumatismo e cerca de $26 \%$ eram tabagistas. Quanto à presença de co-morbidades, pouco mais de um terço dos indivíduos apresentava hipertensão arterial sistêmica, 7,5\% diagnóstico médico de diabetes mellitus e 10,1\% angina pectoris. Além disto, excesso de peso e sedentarismo foram verificados em mais de $50 \%$ dos entrevistados.

A prevalência geral de sintomas articulares crônicos foi de 36,5\% (IC95\%: 34,7-38,2), e as mulheres apresentaram uma prevalência 1,5 (IC95\%: 1,3-1,6) vez maior que os homens. Na faixa etária dos 20 a 29 anos, $19 \%$ dos indivíduos relataram sintomas articulares crônicos e uma prevalência 2,9 (IC95\%: 2,4-3,5) vezes maior foi encontrada naqueles com 60 anos ou mais ( $<<0,001)$. Não houve diferença, conforme a cor da pele, pa- 
Descrição da amostra e da prevalência de sintomas articulares crônicos de acordo com as variáveis independentes estudadas. Pelotas, Rio Grande do Sul, Brasil ( $n=2.953)$.

\begin{tabular}{|c|c|c|c|c|c|}
\hline \multirow[t]{2}{*}{ Variáveis } & \multicolumn{2}{|c|}{ Geral } & \multicolumn{3}{|c|}{ Sitomas articulares crônicos } \\
\hline & $\mathrm{n}$ & $\%$ & Prevalência (\%) & IC95\% & Valor de $p$ \\
\hline Sexo & & & & & $<0,001$ * \\
\hline Feminino & 1.679 & 56,9 & 42,4 & $39,6-45,3$ & \\
\hline Masculino & 1.274 & 43,1 & 28,7 & $25,9-31,6$ & \\
\hline Idade (anos) & & & & & $<0,001 * *$ \\
\hline $20-29$ & 710 & 24,1 & 19,0 & $16,1-22,4$ & \\
\hline $30-39$ & 550 & 18,6 & 27,6 & $23,8-31,9$ & \\
\hline $40-49$ & 593 & 20,1 & 38,5 & $34,3-42,8$ & \\
\hline $50-59$ & 517 & 17,5 & 46,4 & $41,9-51,1$ & \\
\hline$\geq 60$ & 583 & 19,7 & 55,3 & $50,6-59,8$ & \\
\hline Cor & & & & & 0,927 * \\
\hline Branca & 2.229 & 75,6 & 36,7 & $34,3-39,2$ & \\
\hline Negra/Parda & 608 & 20,6 & 35,9 & $31,2-40,8$ & \\
\hline Outras & 112 & 3,8 & 35,7 & $26,7-45,9$ & \\
\hline Escolaridade (anos completos) & & & & & $<0,001$ ** \\
\hline $0-4$ & 685 & 23,2 & 51,1 & $46,7-55,5$ & \\
\hline $5-8$ & 932 & 31,6 & 40,3 & $36,7-43,9$ & \\
\hline $9-12$ & 833 & 28,2 & 27,0 & $23,7-30,7$ & \\
\hline$\geq 13$ & 502 & 17,0 & 24,9 & $21,7-28,4$ & \\
\hline História familiar de artrite & & & & & $<0,001$ * \\
\hline Não & 1.515 & 54,5 & 31,2 & $28,3-34,1$ & \\
\hline Sim & 1.266 & 45,5 & 41,6 & $38,6-44,6$ & \\
\hline Tabagismo & & & & & $<0,001$ * \\
\hline Nunca fumou & 1.545 & 52,3 & 33,0 & $30,4-35,6$ & \\
\hline Ex-tabagista & 647 & 21,9 & 45,2 & $40,9-49,5$ & \\
\hline Tabagista atual & 761 & 25,8 & 36,1 & $32,3-40,2$ & \\
\hline Hipertensão arterial sistêmica & & & & & $<0,001$ * \\
\hline Não & 1.840 & 62,9 & 30,3 & $27,9-32,9$ & \\
\hline Sim & 1.086 & 37,1 & 47,2 & $43,9-50,6$ & \\
\hline Diabetes mellitus & & & & & $<0,001$ * \\
\hline Não & 2.718 & 92,5 & 34,5 & $32,3-36,8$ & \\
\hline Sim & 221 & 7,5 & 60,9 & $53,8-67,6$ & \\
\hline 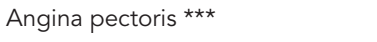 & & & & & $<0,001$ * \\
\hline Não & 1.522 & 89,9 & 44,1 & $41,3-47,0$ & \\
\hline Sim & 171 & 10,1 & 69,0 & $60,2-76,7$ & \\
\hline Índice de massa corporal (kg/m²) & & & & & $<0,001$ ** \\
\hline$\leq 24,9$ & 1.283 & 48,0 & 28,5 & $25,6-31,5$ & \\
\hline $25-29,9$ & 947 & 35,4 & 38,4 & $35,4-41,6$ & \\
\hline$\geq 30$ & 445 & 16,6 & 48,0 & $42,5-53,5$ & \\
\hline Sedentarismo & & & & & $0,421 *$ \\
\hline Não & 1.421 & 48,4 & 35,7 & $32,9-38,6$ & \\
\hline Sim & 1.518 & 51,6 & 37,1 & $34,2-40,0$ & \\
\hline
\end{tabular}

Nota: IMC foi a variável com o maior percentual de valores ignorados (9,4\%); as demais tiveram entre $0,03 \%$ a $0,9 \%$.

* $\chi^{2}$ para heterogeneidade;

** $\chi^{2}$ para tendência linear;

$\star \star *$ Indivíduos com idade $\geq 40$ anos $(n=1.693)$. 
ra a presença de sintomas articulares crônicos ( $p=0,927$ ). Comparados aos indivíduos com 13 anos ou mais de escolaridade, aqueles com até quatro anos apresentaram praticamente duas (IC95\%: 1,6-2,3) vezes mais sintomas articulares crônicos $(\mathrm{p}<0,001)$. Quanto à história familiar de artrite, a prevalência de sintomas articulares crônicos foi $30 \%$ maior naqueles com tal histórico, comparados aos demais $(\mathrm{p}<0,001)$. Entre os adictos do tabaco no passado, os sintomas articulares crônicos foram mais relatados do que naqueles sem este vício, com prevalências de $45,2 \%$ e $33 \%$, respectivamente $(\mathrm{p}<0,001)$. Os indivíduos com hipertensão arterial sistêmica, diabetes mellitus e angina pectoris apresentaram prevalências de sintomas articulares crônicos, no mínimo 60\% maiores do que aqueles sem estas co-morbida$\operatorname{des}(\mathrm{p}<0,001)$. Comparados às pessoas com IMC normal, a prevalência de sintomas articulares crônicos foi $35 \%$ e $69 \%$ maior nos entrevistados com sobrepeso e obesidade, respectivamente $(p<0,001)$. Sintomas articulares crônicos se distribuíram de forma semelhante entre os indivíduos sedentários e não sedentários ( $\mathrm{p}=0,421$ ).

A Figura 1 mostra a distribuição dos diferentes sintomas articulares crônicos de forma estratificada por sexo. No sexo masculino, a prevalência de pelo menos um sintoma foi de $28,7 \%$ (IC95\%: 25,9-31,6), enquanto, no sexo feminino, foi de 42,4\% (IC95\%: 39,6-45,3). No entanto, a presença da tríade - dor, edema e rigidez articulares - apresentou uma prevalência de 14,1\% (IC95\%: 12,2-16,2) nas mulheres e 5,5\% (IC95\%: $4,3-7,0)$ nos homens. Isoladamente, dor articular foi o sintoma mais freqüentemente relatado, em ambos os sexos, com prevalência média de 11,9\% (IC95\%: 10,7-13,2).

Na Tabela 2, estão apresentadas a análise bruta e ajustada para o sexo masculino, com as razões de prevalência para o relato de sintomas articulares crônicos, seus respectivos IC95\% e valores de p. Na análise bruta, observa-se que há um aumento linear da prevalência de sintomas articulares crônicos à medida que aumenta a idade $(\mathrm{p}<0,001)$. Por outro lado, com o aumento da escolaridade há um decréscimo da prevalência de sintomas articulares crônicos, e entre os homens com até quatro anos de estudo a prevalência é mais do que o dobro do que naqueles com 13 anos ou mais de escolaridade. A presença de história familiar de artrite ou reumatismo não revelou associação significativa com o desfecho ( $p=0,102)$. Em comparação aos não fumantes, os homens com passado de tabagismo apresentaram prevalências maiores de sintomas articulares crônicos $(\mathrm{p}<0,001)$. Aqueles que possuíam hipertensão arterial sistêmica, diabetes mellitus e angina apresentaram uma prevalência $41 \%$,

\section{Figura 1}

Freqüência dos diferentes sintomas articulares crônicos de acordo com o sexo. Pelotas, Rio Grande do Sul, Brasil, 2007

1a) Sexo feminino $(n=1.679)$
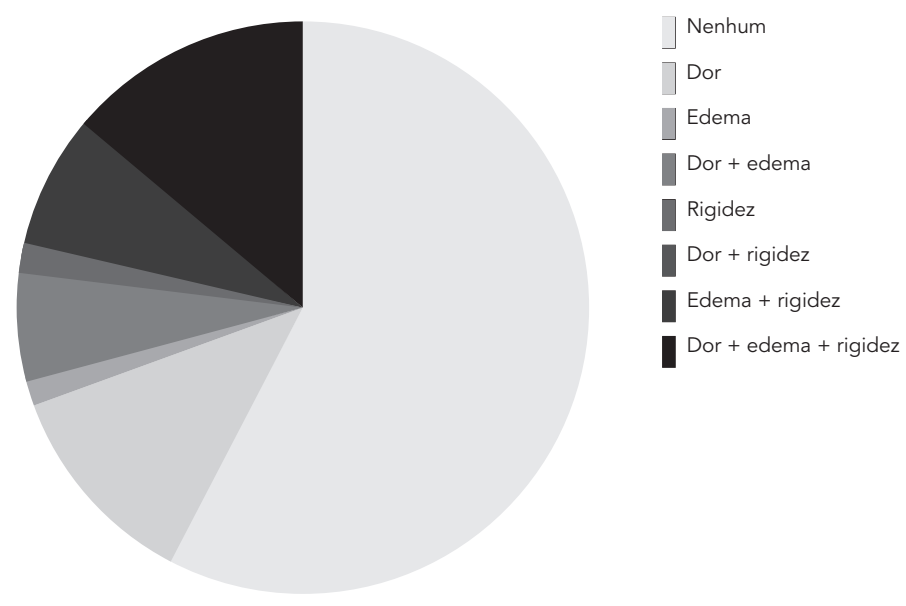

1b) Sexo masculino $(n=1.274)$.
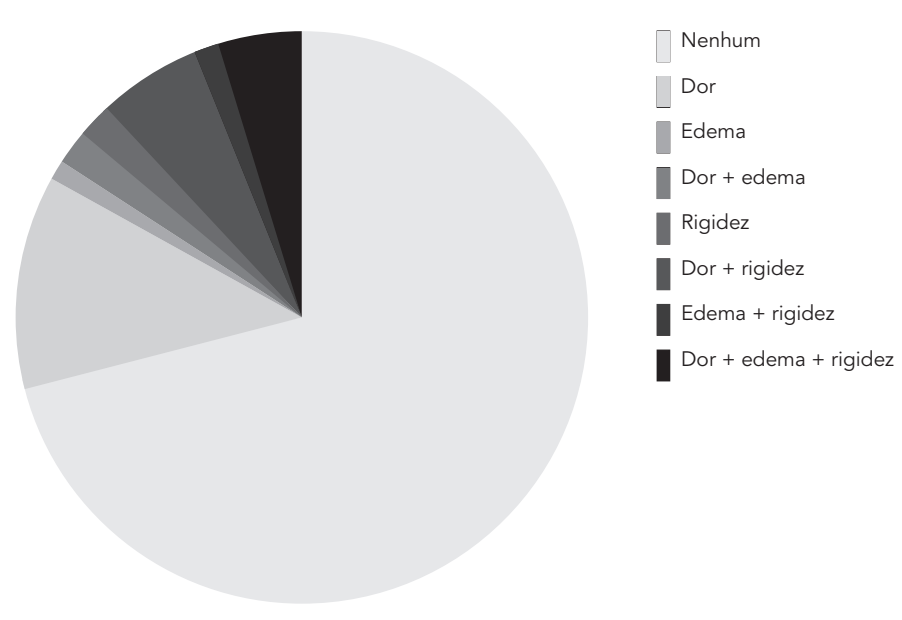

$48 \%$ e $39 \%$ maior de sintomas articulares crônicos, respectivamente, quando comparados aos sem estes diagnósticos. Por fim, ter sobrepeso acarretou um aumento de $13 \%$ na prevalência de sintomas articulares crônicos e ter obesidade $61 \%$, quando comparados aos indivíduos com IMC normal ( $\mathrm{p}<0,001)$. Na análise ajustada, a partir da faixa etária dos 40-49 anos, a prevalência de sintomas articulares crônicos, passa a ser mais que o dobro daquela encontrada nos ho- 
Tabela 2

Razões de prevalências brutas e ajustadas de sintomas articulares crônicos no sexo masculino de acordo com as variáveis independentes. Pelotas, Rio Grande do Sul, Brasil, 2007 ( $n=1.274)$.

\begin{tabular}{|c|c|c|c|c|c|}
\hline \multirow[t]{2}{*}{ Nível } & \multirow[t]{2}{*}{ Variáveis } & \multicolumn{2}{|c|}{ Bruta } & \multicolumn{2}{|c|}{ Ajustada } \\
\hline & & RP (IC95\%) & Valor de $p$ & RP (IC95\%) & Valor de $p$ \\
\hline \multirow[t]{10}{*}{1} & Idade (anos) & & $<0,001$ * & & $<0,001$ * \\
\hline & $20-29$ & 1,00 & & 1,00 & \\
\hline & $30-39$ & $1,29(0,92-1,79)$ & & $1,29(0,92-1,79)$ & \\
\hline & $40-49$ & $2,07(1,53-2,81)$ & & $2,07(1,53-2,81)$ & \\
\hline & $50-59$ & $2,16(1,61-2,90)$ & & $2,16(1,61-2,90)$ & \\
\hline & $\geq 60$ & $2,64(1,93-3,62)$ & & $2,64(1,93-3,62)$ & \\
\hline & Cor & & 0,716 ** & & 0,892 ** \\
\hline & Branca & 1,00 & & 1,00 & \\
\hline & Negra/Parda & $0,90(0,69-1,16)$ & & $0,96(0,75-1,24)$ & \\
\hline & Outras & $0,98(0,56-1,72)$ & & $1,11(0,63-1,94)$ & \\
\hline \multirow[t]{8}{*}{2} & Escolaridade (anos completos) & & $<0,001 *$ & & $<0,001$ * \\
\hline & $0-4$ & $2,45(1,85-3,24)$ & & $2,15(1,58-2,92)$ & \\
\hline & $5-8$ & $1,73(1,31-2,28)$ & & $1,75(1,31-2,33)$ & \\
\hline & $9-12$ & $1,23(0,88-1,73)$ & & $1,43(1,03-2,00)$ & \\
\hline & $\geq 13$ & 1,00 & & 1,00 & \\
\hline & História familiar de artrite & & 0,102 ** & & 0,030 ** \\
\hline & Não & 1,00 & & 1,00 & \\
\hline & Sim & $1,17(0,97-1,40)$ & & $1,24(1,02-1,50)$ & \\
\hline \multirow[t]{13}{*}{3} & Tabagismo & & $0,001 \star \star \star$ & & $0,004 * \star$ \\
\hline & Nunca & 1,00 & & 1,00 & \\
\hline & Ex-tabagista & $1,86(1,54-2,24)$ & & $1,45(1,17-1,80)$ & \\
\hline & Tabagista atual & $1,44(1,12-1,83)$ & & $1,24(0,96-1,60)$ & \\
\hline & Hipertensão arterial sistêmica & & $<0,001 \star \star$ & & $0,415 * \star$ \\
\hline & Não & 1,00 & & 1,00 & \\
\hline & Sim & $1,41(1,18-1,68)$ & & $1,09(0,89-1,34)$ & \\
\hline & Diabetes mellitus & & 0,004 ** & & $0,897 * \star$ \\
\hline & Não & 1,00 & & 1,00 & \\
\hline & Sim & $1,48(1,13-1,92)$ & & $1,02(0,76-1,37)$ & \\
\hline & Angina $* \star \star$ & & 0,035 ** & & 0,161 ** \\
\hline & Não & 1,00 & & 1,00 & \\
\hline & Sim & $1,39(1,02-1,89)$ & & $1,27(0,91-1,79)$ & \\
\hline \multirow[t]{7}{*}{4} & Índice de massa corporal (kg/m²) & & $<0,001 *$ & & 0,001 * \\
\hline & $\leq 24,9$ & 1,00 & & 1,00 & \\
\hline & $25-29,9$ & $1,13(0,95-1,35)$ & & $1,12(0,91-1,36)$ & \\
\hline & $\geq 30$ & $1,61(1,28-2,02)$ & & $1,59(1,26-2,01)$ & \\
\hline & Sedentarismo & & 0,172 * & & 0,847 * \\
\hline & Não & 1,00 & & 1,00 & \\
\hline & Sim & $1,13(0,95-1,34)$ & & $1,02(0,84-1,23)$ & \\
\hline
\end{tabular}

Nota: o maior percentual de valores ignorados foi para a variável história familiar de artrite (6,8\%); as demais tiveram entre 0,1\% a 4,2\%.

* Teste de Wald para tendência;

** Teste de Wald para heterogeneidade;

$\star \star \star$ Somente indivíduos com idade $\geq 40$ anos $(n=704)$. 
mens com 20 a 29 anos ( $p<0,001)$. A presença de sintomas articulares crônicos manteve uma associação inversa com alta escolaridade $(\geq 13$ anos) mesmo após ajuste para a idade $(\mathrm{p}<0,001)$. Por outro lado, relato de história familiar de artrite que não estava associado a sintomas articulares crônicos, na análise bruta, mostrou uma prevalência $24 \%$ maior quando comparados àqueles sem este antecedente familiar ( $\mathrm{p}=0,030$ ). Quanto à presença das co-morbidades hipertensão arterial sistêmica, diabetes mellitus e angina, houve perda da associação estatisticamente significativa com sintomas articulares crônicos após o ajuste para idade, escolaridade, história familiar de artrite e tabagismo, no sexo masculino. O IMC se manteve, independentemente, associado ao relato de sintomas articulares crônicos e, a prevalência deles aumentou linearmente com o aumento do IMC ( $p<0,001)$. Todavia, os sintomas articulares crônicos não se distribuíram diferentemente entre os sedentários ou não.

Para o sexo feminino, na análise bruta (Tabela 3), ocorreu aumento direto com efeito dose-resposta da presença de sintomas articulares crônicos em relação à idade $(\mathrm{p}<0,001)$. Quanto à escolaridade, aquelas mulheres com até quatro anos de estudo tiveram uma prevalência $86 \%$ maior de sintomas articulares crônicos comparadas às com 13 anos ou mais de escolaridade. Quando comparados aos indivíduos sem história familiar de artrite ou reumatismo, os que possuíam este antecedente apresentaram uma prevalência 1,3 vez maior do desfecho $(p<0,001)$. O fato de ser ex-tabagista acarretou um acréscimo na prevalência de sintomas articulares crônicos, comparadas às não tabagistas. Mulheres com hipertensão arterial sistêmica, diabetes mellitus e angina apresentaram prevalências, no mínimo 1,6 vez maior do que aquelas sem tais co-morbidades. Quanto ao IMC, encontrou-se aumento linear da prevalência de sintomas articulares crônicos com o aumento do IMC ( $p<0,001)$. Na análise ajustada, quando comparadas às mulheres com 20 a 29 anos, aquelas com idade entre 30 a 39 anos apresentaram uma prevalência 1,6 vez maior e, as com idade $\geq 60$ anos, 2,9 vezes maior. À medida que aumentaram os anos de escolaridade, diminuiu linearmente o risco de sintomas articulares crônicos $(\mathrm{p}<0,001)$. História familiar de artrite também se manteve associada a sintomas articulares crônicos, com uma prevalência 1,4 vez maior nas mulheres com este histórico, mesmo após ajuste para fatores de confusão ( $\mathrm{p}<0,001)$. Da mesma maneira, as mulheres extabagistas apresentaram prevalência $22 \%$ maior de sintomas articulares crônicos, quando comparadas às não tabagistas $(\mathrm{p}=0,007)$. Quanto à presença de co-morbidades, a presença de hi- pertensão arterial sistêmica, diabetes mellitus e angina associou-se, de maneira independente, a um acréscimo na prevalência de sintomas articulares crônicos de $18 \%$ e $40 \%$, respectivamente. Comparadas às mulheres com IMC normal, ter sobrepeso representou um acréscimo de $34 \%$ na prevalência de sintomas articulares crônicos e obesidade, $42 \%$, mantendo o efeito dose-resposta $(\mathrm{p}<0,001)$. Cor da pele e sedentarismo não se mostraram associados a sintomas articulares crônicos.

Diagnóstico médico de artrite ou reumatismo foi relatado por 78 homens $(6,2 \%)$ e por $303 \mathrm{mu}-$ lheres $(18,2 \%)$. Com relação ao trabalho, 21,5\% dos homens e $11,7 \%$ das mulheres referiram desempenhar trabalho braçal sempre, nos últimos 24 meses, enquanto $63,6 \%$ e $65 \%$ dos homens e mulheres, respectivamente, desempenharam trabalho repetitivo sempre, neste mesmo período. Quanto à prevalência de restrições secundárias a sintomas articulares crônicos, 12,6\% (IC95\%: 9,616,4) dos homens e 16,2\% (IC95\%: 13,9-18,9) das mulheres relataram muita restrição para as atividades da vida diária. Assim como 19,7\% (IC95\%: 16,2-23,7) dos homens e 21,5\% (IC95\%: 18,7-24,5) das mulheres referiram sentirem-se muito restritos para trabalhar e/ou estudar (resultados não apresentados nas tabelas).

O efeito do delineamento encontrado para o desfecho - sintomas articulares crônicos - foi de 1,8 e o coeficiente de correlação intraclasse foi de 0,08 . O kappa foi de 0,6 para o relato de diagnóstico médico de artrite ou reumatismo.

\section{Discussão}

Este é o primeiro estudo transversal brasileiro de base populacional realizado com objetivo específico de avaliar sintomas articulares crônicos em indivíduos a partir dos 20 anos de idade. Mendoza-Sassi et al. 15 avaliaram a presença de vários sinais e sintomas auto-referidos em indivíduos com idade $\geq 15$ anos e encontrou uma prevalência geral de 35,6\% para dor nas juntas; não houve, no entanto, diferenciação quanto ao tempo de duração dos sintomas, o que fornece uma estimativa conjunta para dores agudas, subagudas e crônicas. Outro ponto que cabe salientar é que a presença de edema e rigidez articular não foi abordada no estudo de Mendoza-Sassi et al. 15 . Como sintomas articulares agudos ou subagudos não eram nosso foco de interesse, definimos que a sintomatologia articular deveria estar presente na maioria dos dias e por, no mínimo, 45 dias. Esta definição de tempo pode sofrer variações na literatura, porque não se trata de uma doença específica, como osteoartrose ou artrite 
Tabela 3

Razões de prevalências brutas e ajustadas de sintomas articulares crônicos no sexo feminino de acordo com as variáveis independentes. Pelotas, Rio Grande do Sul, Brasil, 2007 ( $n=1.679)$.

\begin{tabular}{|c|c|c|c|c|c|}
\hline \multirow[t]{2}{*}{ Nível } & \multirow[t]{2}{*}{ Variáveis } & \multicolumn{2}{|c|}{ Bruta } & \multicolumn{2}{|c|}{ Ajustada } \\
\hline & & RP (IC95\%) & Valor de p & RP (IC95\%) & Valor de $p$ \\
\hline \multirow[t]{10}{*}{1} & Idade (anos) & & $<0,001 *$ & & $<0,001 *$ \\
\hline & $20-29$ & 1,00 & & 1,00 & \\
\hline & $30-39$ & $1,55(1,21-1,98)$ & & $1,55(1,21-1,98)$ & \\
\hline & $40-49$ & $1,97(1,59-2,45)$ & & $1,97(1,59-2,45)$ & \\
\hline & $50-59$ & $2,57(2,09-3,16)$ & & $2,57(2,09-3,16)$ & \\
\hline & $\geq 60$ & $2,94(2,36-3,66)$ & & $2,94(2,36-3,66)$ & \\
\hline & Cor & & 0,816 ** & & 0,513 ** \\
\hline & Branca & 1,00 & & 1,00 & \\
\hline & Negra/Parda & $1,04(0,90-1,20)$ & & $1,08(0,94-1,24)$ & \\
\hline & Outras & $0,96(0,72-1,27)$ & & $0,99(0,77-1,27)$ & \\
\hline \multirow[t]{8}{*}{2} & Escolaridade (anos completos) & & $<0,001$ * & & $<0,001$ * \\
\hline & $0-4$ & $1,86(1,55-2,22)$ & & $1,40(1,16-1,69)$ & \\
\hline & $5-8$ & $1,60(1,35-1,90)$ & & $1,48(1,25-1,75)$ & \\
\hline & $9-12$ & $1,02(0,82-1,27)$ & & $1,11(0,90-1,36)$ & \\
\hline & $\geq 13$ & 1,00 & & 1,00 & \\
\hline & História familiar de artrite & & $<0,001 \star \star$ & & $<0,001 \star \star$ \\
\hline & Não & 1,00 & & 1,00 & \\
\hline & Sim & $1,34(1,20-1,50)$ & & $1,36(1,22-1,53)$ & \\
\hline \multirow[t]{13}{*}{3} & Tabagismo & & 0,002 ** & & 0,008 ** \\
\hline & Nunca & 1,00 & & 1,00 & \\
\hline & Ex-tabagista & $1,27(1,11-1,46)$ & & $1,22(1,08-1,39)$ & \\
\hline & Tabagista atual & $1,03(0,90-1,19)$ & & $1,12(0,96-1,30)$ & \\
\hline & Hipertensão arterial sistêmica & & 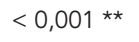 & & $<0,012$ ** \\
\hline & Não & 1,00 & & 1,00 & \\
\hline & Sim & $1,67(1,50-1,85)$ & & $1,18(1,04-1,36)$ & \\
\hline & Diabetes mellitus & & $<0,001 \star \star$ & & 0,018 ** \\
\hline & Não & 1,00 & & 1,00 & \\
\hline & Sim & $1,78(1,58-2,00)$ & & $1,18(1,03-1,36)$ & \\
\hline & Angina $\star \star \star$ & & $<0,001 \star \star$ & & $<0,001$ ** \\
\hline & Não & 1,00 & & 1,00 & \\
\hline & Sim & $1,56(1,37-1,77)$ & & $1,40(1,23-1,61)$ & \\
\hline \multirow[t]{7}{*}{4} & Índice de massa corporal (kg/m²) & & $<0,001$ * & & $<0,001$ * \\
\hline & $\leq 24,9$ & 1,00 & & 1,00 & \\
\hline & $25-29,9$ & $1,58(1,36-1,82)$ & & $1,34(1,16-1,55)$ & \\
\hline & $\geq 30$ & $1,76(1,46-2,11)$ & & $1,42(1,17-1,71)$ & \\
\hline & Sedentarismo & & 0,585 ** & & 0,175 ** \\
\hline & Não & 1,00 & & 1,00 & \\
\hline & Sim & $0,97(0,86-1,09)$ & & $0,91(0,80-1,04)$ & \\
\hline
\end{tabular}

Nota: o maior percentual de valores ignorados foi para a variável índice de massa corporal $(13,4 \%)$; as demais tiveram entre $0,1 \%$ a $6,6 \%$.

* Teste de Wald para tendência;

** Teste de Wald para heterogeneidade;

$\star \star \star$ Indivíduos com idade $\geq 40$ anos $(n=989)$. 
reumatóide, mas sim de um grupo heterogêneo de enfermidades com sintomas comuns. Assim sendo, consideramos que ao determinarmos um período de seis semanas para a duração dos sintomas articulares, e não de quatro como os estudos americanos, havendo um erro de classificação, este seria conservador, pois iria subestimar a prevalência de sintomas articulares crônicos. O pequeno número de perdas e recusas $(6,2 \%)$ e a comparabilidade da amostra estudada com a distribuição dos dados do IBGE (censo 2000) quanto a sexo, faixas etárias e condições sócioeconômicas, entre outras variáveis, evidenciam que os resultados encontrados provavelmente são representativos da população adulta de Pelotas.

Alguns aspectos devem ser considerados na interpretação dos resultados deste estudo. Um deles é a possibilidade de viés de memória, que foi minimizado em virtude do período recordatório ser restrito aos últimos 12 meses. $\mathrm{O}$ índice de massa corporal foi calculado com base no relato de peso e altura dos entrevistados e isso poderia produzir uma estimativa errônea, conseqüentemente, alterando as associações encontradas. Entretanto, há evidências na literatura de que o peso referido apresenta boa correlação com o peso medido 16; além disto, foi realizada uma validação de peso e altura em uma subamostra - de 300 indivíduos - e encontrado um elevado coeficiente de concordância (kappa ponderado de 0 ,8). Uma vez que o delineamento utilizado não permite inferir temporalidade, não se pode afastar causalidade reversa na associação entre excesso de peso e sintomas articulares crônicos.

Certamente a avaliação médica dos indivíduos com queixas articulares crônicas é mais precisa do que o auto-relato, todavia, não é factível para rastreamento populacional. Além do que, a acurácia do relato de condições reumatológicas quando comparadas aos dados de prontuários médicos, apresenta uma boa concordância geral $-87 \%$, segundo a literatura 17 .

A prevalência média de sintomas articulares crônicos encontrada neste estudo foi semelhante àquela encontrada em norte-americanos, com equivalente faixa etária 4 . $\mathrm{O}$ achado de uma prevalência maior de sintomas articulares crônicos nas mulheres é unânime na literatura, e as diferenças e variações no perfil de hormônios sexuais femininos são uma parte importante de tal determinação 18,19. Com o avanço da idade, a prevalência de sintomas articulares crônicos aumentou, independentemente do sexo, mas é importante salientar que na faixa etária dos 20 aos 29 anos, 16,3\% (IC95\%: 12,4-21,1) dos homens e 21,4\% (IC95\%: 17,4-25,9) das mulheres apresentaram sintomas articulares crônicos.
Não há consenso na literatura sobre a associação entre cor da pele e sintomas articulares crônicos ou artrite. No presente estudo, independentemente do sexo, cor da pele não apresentou associação com a presença de sintomas articulares crônicos. Contudo, um estudo norte-americano evidenciou que, embora a prevalência de sintomas articulares ou artrite fosse similar em brancos e negros, esses apresentaram mais limitações funcionais secundárias à artrite do que os brancos $(44,2 \%$ vs. $34,1 \%) 20$.

A presença de sintomas articulares crônicos mostra-se consistentemente associada ao menor nível de escolaridade; isso está possivelmente relacionado a piores condições sócio-econômicas e condições de saúde, especialmente em países com grandes desigualdades sociais, como o Brasil. Tais achados evidenciam que os indicadores sócio-econômicos devem ser cuidadosamente considerados antes de inferirmos que eles se devem a cor da pele ou etnia dos indivíduos.

No sexo masculino, a história familiar de artrite isoladamente não apresentou associação estatisticamente significativa com sintomas articulares crônicos, mas quando foram consideradas a idade e a escolaridade dos indivíduos, ela mostrou-se associada ao desfecho, um outro exemplo típico, de confusão negativa.

O papel do tabaco na patogênese das artropatias crônicas, especialmente na artrite reumatóide, parece ser multifatorial. O cigarro tanto pode aumentar o risco de artrite reumatóide mediante a interação com fatores de risco genéticos 21, como pode induzir à inflamação e ao dano articular, por mecanismo imunobiológico, que uma vez desencadeado não cessa com o abandono do vício 22. Estas são algumas das possíveis explicações para a associação encontrada entre sintomas articulares crônicos e tabagismo, em que os ex-fumantes apresentam um maior risco se comparados aos não tabagistas.

Quanto à presença de hipertensão arterial sistêmica, diabetes mellitus ou angina pectoris que no sexo masculino se mostraram associadas a sintomas articulares crônicos na análise bruta, com o ajuste para fatores de risco, evidencia-se que a associação com o desfecho era, provavelmente, por causa do avanço da idade e da menor escolaridade. Por outro lado, no sexo feminino, estas co-morbidades se mantêm associadas tanto na análise bruta quanto na ajustada. Uma explicação possível para este achado é que os mecanismos envolvidos na patogênese dessas doenças sejam realmente diferentes entre homens e mulheres.

A associação de sobrepeso e obesidade com sintomas articulares crônicos e artrite já foi descrita em outros estudos 9,23. É plausível crer que 
o excesso de peso determine a presença de sintomas articulares crônicos em razão não somente de fatores biomecânicos como as propriedades pró-inflamatórias de algumas adipocitocinas. Este último fator já teve seu papel demonstrado na artrite reumatóide 24 .

Cabe ainda salientar que somente $27 \%$ das pessoas com sintomas articulares crônicos têm diagnóstico médico de artrite ou reumatismo. Dentre eles, os homens são ainda menos diagnosticados, apenas $13,8 \%$ versus $33,9 \%$ nas mulheres. $\mathrm{O}$ achado talvez reflita a menor utilização dos serviços de saúde pelos jovens e homens. Corroborando esta hipótese, um estudo norteamericano 25 , de âmbito nacional, com indivíduos de 18 anos ou mais evidenciou que dos adultos com relato de artrite, $16,4 \%$ nunca foram avaliados por um médico. Este grupo de pessoas era prevalentemente de homens e jovens. Aqueles com melhor autopercepção de saúde, menos limitações para o trabalho e sem excesso de peso também tinham menor probabilidade de consultar o médico por conta da artrite.

Trabalho braçal e repetitivo, fortemente relacionados 26,27,28 com uma das mais prevalentes condições reumatológicas - a osteoartrose apresentaram maiores prevalências nos jovens (20 a 29 anos) e menos escolarizados ( $\leq 8$ anos). Isso nos faz refletir sobre a necessidade de investir em programas específicos para esta parcela da população. Novas propostas para diagnosticar precocemente as artropatias crônicas apresentam uma relação direta com a prevenção de incapacidades funcionais e laborais. Em nosso estudo, $15 \%$ dos entrevistados referiram muita limitação gerada pelos sintomas articulares crônicos para atividades de vida diária e 20,5\% para trabalhar ou estudar. Este achado é semelhante ao encontrado para sintomas articulares crônicos em idosos 6 e fornece uma estimativa aproximada do impacto social secundário às enfermidades reumatológicas. Em 1999, nos Estados Unidos, aproximadamente 44 milhões de adultos com 18 anos ou mais apresentavam incapacidade; 7,2 milhões (16,4\%) eram secundárias à artrite ou reumatismo 10 .

Os resultados deste estudo permitem concluir que sintomas articulares crônicos apresentam alta prevalência em adultos e não somente em idosos. Além disso, foi possível identificar fatores de risco não modificáveis como a idade, como também outros fatores potencialmente modificáveis, tais como tabagismo e obesidade. Ainda é necessário que outros estudos avaliem este tipo de abordagem para rastreamento e diagnóstico precoce de doenças articulares crônicas prevenindo assim incapacidades, uma vez que parcela importante da população - homens e jovens - é subdiagnosticada. 


\section{Resumo}

Com o objetivo de estimar a prevalência de sintomas articulares crônicos e seus determinantes, foi realizado um estudo transversal, com 2.953 adultos com 20 anos ou mais, residentes em Pelotas, Rio Grande do Sul, Brasil. Foram investigadas a presença de dor, edema ou rigidez articular, além de informações demográficas, sócio-econômicas e sobre problemas de saúde. A análise multivariável foi realizada pela regressão de Poisson. A prevalência geral de sintomas articulares crônicos foi de 36,5\% (42,4\% nas mulheres e 28,7\% nos homens). A presença da tríade - dor, edema e rigidez articulares - foi de 14,1\% e 5,5\% nas mulheres e homens, respectivamente. Sintomas articulares crônicos se mostraram linearmente associados ao aumento da idade (RP: 2,9; IC95\%: 2,4-3,5), do índice de massa corporal (RP: 1,6; IC95\%: 1,3-2,0) e à menor escolaridade (RP: 1,5; IC95\%: 1,3-1,8). Somente $6 \%$ dos homens e 18\% das mulheres relataram diagnóstico médico de artrite. As prevalências gerais de limitações para atividades de vida diária e para atividades laborais, secundárias a sintomas articulares crônicos, foi de 15\% e $21 \%$, respectivamente. Os jovens e homens representam a maioria dos indivíduos com sintomas articulares crônicos subdiagnosticados.

Artralgia; Artrite; Doenças Reumáticas; Articulações

\section{Colaboradores}

V. R. L. Silva executou a concepção do projeto, trabalho de campo, análise e interpretação dos dados, a redação do artigo e revisão crítica do conteúdo intelectual e aprovou a versão final a ser publicada. A. M. B. Menezes orientou a concepção do projeto, análise e interpretação dos dados, a redação do artigo e a revisão crítica do conteúdo intelectual, além de dar a aprovação final da versão a ser publicada. R. B. Noal co-orientou a concepção do projeto, análise e interpretação dos dados, a redação do artigo e a revisão crítica do conteúdo intelectual, além de dar aprovação final da versão a ser publicada.

\section{Referências}

1. Badley EM, Tennant A. Impact of disablement due to rheumatic disorders in a British population: estimates of severity and prevalence from the Calderdale Rheumatic Disablement Survey. Ann Rheum Dis 1993; 52:6-13.

2. Kelsey JL, Hochberg MC. Epidemiology of chronic musculoskeletal disorders. Annu Rev Public Health 1988; 9:379-401.

3. Reynolds DL, Chambers LW, Badley EM, Bennett KJ, Goldsmith CH, Jamieson E, et al. Physical disability among Canadians reporting musculoskeletal diseases. J Rheumatol 1992; 19:1020-30.

4. Centers for Disease Control and Prevention. Prevalence of self-reported arthritis or chronic joint symptoms among adults - United States, 2001. MMWR Morb Mortal Wkly Rep 2002; 51:948-50.
5. Lima-Costa MF, Barreto SM, Giatti L. Condições de saúde, capacidade funcional, uso de serviços de saúde e gastos com medicamentos da população idosa brasileira: um estudo descritivo baseado na Pesquisa Nacional por Amostra de Domicílios. Cad Saúde Pública 2003; 19:735-43.

6. Machado GP, Barreto SM, Passos VM, Lima-Costa MF. Projeto Bambuí: prevalência de sintomas articulares crônicos em idosos. Rev Assoc Med Bras 2004; 50:367-72.

7. Lockshin MD. Invited review: sex ratio and rheumatic disease. J Appl Physiol 2001; 91:2366-73.

8. Badley EM, Kasman NM. The impact of arthritis on Canadian women. BMC Womens Health 2004; 4 Suppl 1:S18. 
9. Centers for Disease Control and Prevention. Prevalence and impact of chronic joint symptoms seven states, 1996. MMWR Morb Mortal Wkly Rep 1998; 47:345-51.

10. Centers for Disease Control and Prevention. Prevalence of disabilities and associated health conditions among adults - United States, 1999. MMWR Morb Mortal Wkly Rep 2001; 50:120-5.

11. Centers for Disease Control and Prevention. Behavioral risk factor surveillance system survey questionnaire. Atlanta: Centers for Disease Control and Prevention, US Department of Health and Human Services; 2001.

12. Craig CL, Marshall AL, Sjöström M, Bauman AE, Booth ML, Ainsworth BE, et al. International physical activity questionnaire: 12-country reliability and validity. Med Sci Sports Exerc 2003; 35: 1381-95.

13. World Health Organization. Physical status: the use and interpretation of anthropometry. Report of WHO Expert Committee. Geneva: World Health Organization; 1995. (Technical Reports Series, 854).

14. Rose GA, Blackburn H. Cardiovascular survey methods. Monogr Ser World Health Organ 1968; 56:1-188.

15. Mendoza-Sassi R, Beria JU, Fiori N, Bortolotto A. Prevalence of signs and symptoms, associated sociodemographic factors and resulting actions in an urban center in southern Brazil. Rev Panam Salud Pública 2006; 20:22-8.

16. Schmidt MI, Duncan BB, Tavares M, Polanczyk CA, Pellanda L, Zimmer PM. Validity of self-reported weight: a study of urban Brazilian adults. Rev Saúde Pública 1993; 27:271-6.

17. Rasooly I, Papageorgiou AC, Badley EM. Comparison of clinical and self reported diagnosis for rheumatology outpatients. Ann Rheum Dis 1995; 54:850-2

18. Karlson EW, Mandl LA, Hankinson SE, Grodstein F. Do breast-feeding and other reproductive factors influence future risk of rheumatoid arthritis? Results from the Nurses' Health Study. Arthritis Rheum 2004; 50:3458-67.
19. Richette P, Corvol M, Bardin T. Estrogens, cartilage, and osteoarthritis. Joint Bone Spine 2003; 70: 257-62.

20. Centers for Disease Control and Prevention. Racial/ethnic differences in the prevalence and impact of doctor-diagnosed arthritis - United States, 2002. MMWR Morb Mortal Wkly Rep 2005; 54: 119-23.

21. Criswell LA, Saag KG, Mikuls TR, Cerhan JR, Merlino LA, Lum RF, et al. Smoking interacts with genetic risk factors in the development of rheumatoid arthritis among older Caucasian women. Ann Rheum Dis 2006; 65:1163-7.

22. Manfredsdottir VF, Vikingsdottir T, Jonsson T, Geirsson AJ, Kjartansson O, Heimisdottir M, et al. The effects of tobacco smoking and rheumatoid factor seropositivity on disease activity and joint damage in early rheumatoid arthritis. Rheumatology (Oxford) 2006; 45:734-40.

23. Mehrotra C, Chudy N, Thomas V. Obesity and physical inactivity among Wisconsin adults with arthritis. WMJ 2003; 102:24-8.

24. Senolt L, Housa D, Vernerova Z, Jirasek T, Svobodova R, Veigl D, et al. Resistin in rheumatoid arthritis synovial tissue, synovial fluid and serum. Ann Rheum Dis 2007; 66:458-63.

25. Rao JK, Callahan LF, Helmick 3rd CG. Characteristics of persons with self-reported arthritis and other rheumatic conditions who do not see a doctor. J Rheumatol 1997; 24:169-73.

26. Cole DC, Ibrahim SA, Shannon HS, Scott F, Eyles J. Work correlates of back problems and activity restriction due to musculoskeletal disorders in the Canadian national population health survey (NPHS) 1994-5 data. Occup Environ Med 2001; 58:728-34

27. Cooper C, Campbell L, Byng P, Croft P, Coggon D. Occupational activity and the risk of hip osteoarthritis. Ann Rheum Dis 1996; 55:680-2.

28. Cooper C, McAlindon T, Coggon D, Egger P, DieppeP. Occupational activity and osteoarthritis of the knee. Ann Rheum Dis 1994; 53:90-3.

Recebido em 01/Abr/2009

Versão final reapresentada em 19/Ago/2009

Aprovado em 08/Set/2009 